\title{
TREATMENT OF HYPERTENSION IN THE ELDERLY PATIENT
}

\author{
S. W. HOOBLER, M.D.*
}

The University of Michigan Medical School, Ann Arbor, Michigan

\section{CLASSIFICATION}

From the standpoint of practical therapeutics, four kinds of hypertension in the elderly should be distinguished:

1. Arteriosclerotic, associated with arteriosclerosis of the large vessels.

This is manifested by a wide pulse pressure and usually has a benign prognosis. Treatment is symptomatic only: salt restriction or mild depressor agents often dramatically relieve symptoms without great effect on the blood pressure.

2. "Malignant" hypertension as manifested by rapidly rising blood pressure, papilledema, retinal hemorrhages and exudates, and renal damage.

This complication of hypertensive disease is rare in the elderly. As pointed out by Perera (1), it may be the clue to a Goldblatt type of hypertension, with one kidney involved by an arteriosclerotic plaque or, as we have seen recently, by a slowly progressing ureteral obstruction such as that caused by carcinoma or irradiation fibrosis.

3. "Essential" hypertension with onset after the age of 50 .

It is most unusual, as Perera has emphasized, for hypertension to begin after the age of 40 . It is probable that hypertension with so late an inception will remain benign. This type is easily confused with the arteriosclerotic variety and is to be identified by a relatively smaller pulse pressure and a diastolic blood pressure usually over 110 . It needs no treatment, unless for symptoms or for vascular complications.

4. "Essential" hypertension with onset in middle life and extending into the later years.

When this variety is identified by a specific history, the prognosis for the individual is largely based upon the magnitude of vascular disease in the heart, (by teleoroentgenogram and electrocardiogram), kidneys (urine albumin, phenolsulfonphthalein, urea or creatinine clearance), and cerebral vessels (history or signs of "strokes," vasospastic episodes, or diffuse cerebral degeneration). If these findings are normal, and particularly if the patient is an obese female, the prognosis appears excellent, almost regardless of the high blood pressure. However, in cases with sustained high pressures, a cerebral hemorrhage may occur without warning. If any complications are present, treatment is justified, but the choice of drug will depend upon the age and general status of the patient. Drastic measures are to be avoided.

\section{TREATMENT}

Treatment may take several forms such as dietary, surgical or pharmaceutical. Salt should be restricted to the $200 \mathrm{mg}$. level for a trial period of three months,

Received for publication October 14, 1953.

* Associate Professor of Internal Medicine. 
during which blood pressure, salt excretion and symptomatology are checked on fortnightly visits to the physician. If improvement does not occur at the end of this time, the diet may be gradually abandoned. If the blood pressure falls or symptoms such as headache, dizziness, angina, dyspnea or edema are relieved, the diet is gradually modified upward to the $500-800 \mathrm{mg}$. level. The usual experience of a patient on the low-salt diet is that lassitude and weight loss occur during a period of from three to four weeks, followed by stabilization and an improved sense of well-being. Consequently, at the end of the trial period, many patients refuse to return to a free salt intake, because they feel better without it. Unfortunately, reduction in blood pressure of significant degree is infrequently observed, but the symptomatic relief and the alleviation or postponement of cardiac symptoms seems to justify this management in most elderly hypertensive subjects. In my experience, cases of excessive sodium loss associated with the "low-salt syndrome" are rarely encountered, although elderly patients with renal function less than 50 per cent of normal may occasionally get into difficulty during such a rigid salt restriction. If further measures, such as diuretics or resins are prescribed for elderly hypertensive individuals without edema, serious derangements may occur.

Sympathectomy has in general been considered inapplicable to the elderly hypertensive patient but, in my opinion, certain cases clearly benefit. The expectation of significant reduction in blood pressure is at least as good as in the younger subjects (2) and significant symptomatic relief may also occur. In most instances, less drastic methods will prove satisfactory in the elderly, in whom our therapeutic objective should not be normalization of the blood pressure but rather relief of symptoms and reduction to reasonable levels, in order to avoid or lessen the complications of the disease.

Drug treatment of hypertensive disease is still in the exploratory stage. Table 1 illustrates the present choice of therapeutic agents. Rauwolfia serpentina derivatives are only mildly effective but some of their side effects may be particularly useful in the management of the elderly (3). Hydrazinophthalazine hydrochloride (hydralazine) (Apresoline) is an excitatory drug which greatly increases the circulation (4). It should be employed cautiously in cardiac patients. Hexamethonium chloride (5) and Veratrum viride (Protoveratrine) derivatives (6) are potent and should be used with great caution in the elderly.

Since hypertensive disease varies widely, an attempt will now be made to relate the treatment to various types of hypertensive disease in the elderly, as follows:

1. Blood pressure usually below 200 systolic; no complications No treatment necessary.

2. Blood pressure usually over 200 systolic; no complications

Treatment here is elective, depending upon the patient's age and cooperation. These individuals run the risk of cerebral hemorrhage at any time, but if in good condition and cooperative, they deserve a trial of therapy, in this order: 1) Sodium restriction, 2) Rauwolfia, 3) Apresoline. 
s. W. HOOBLER

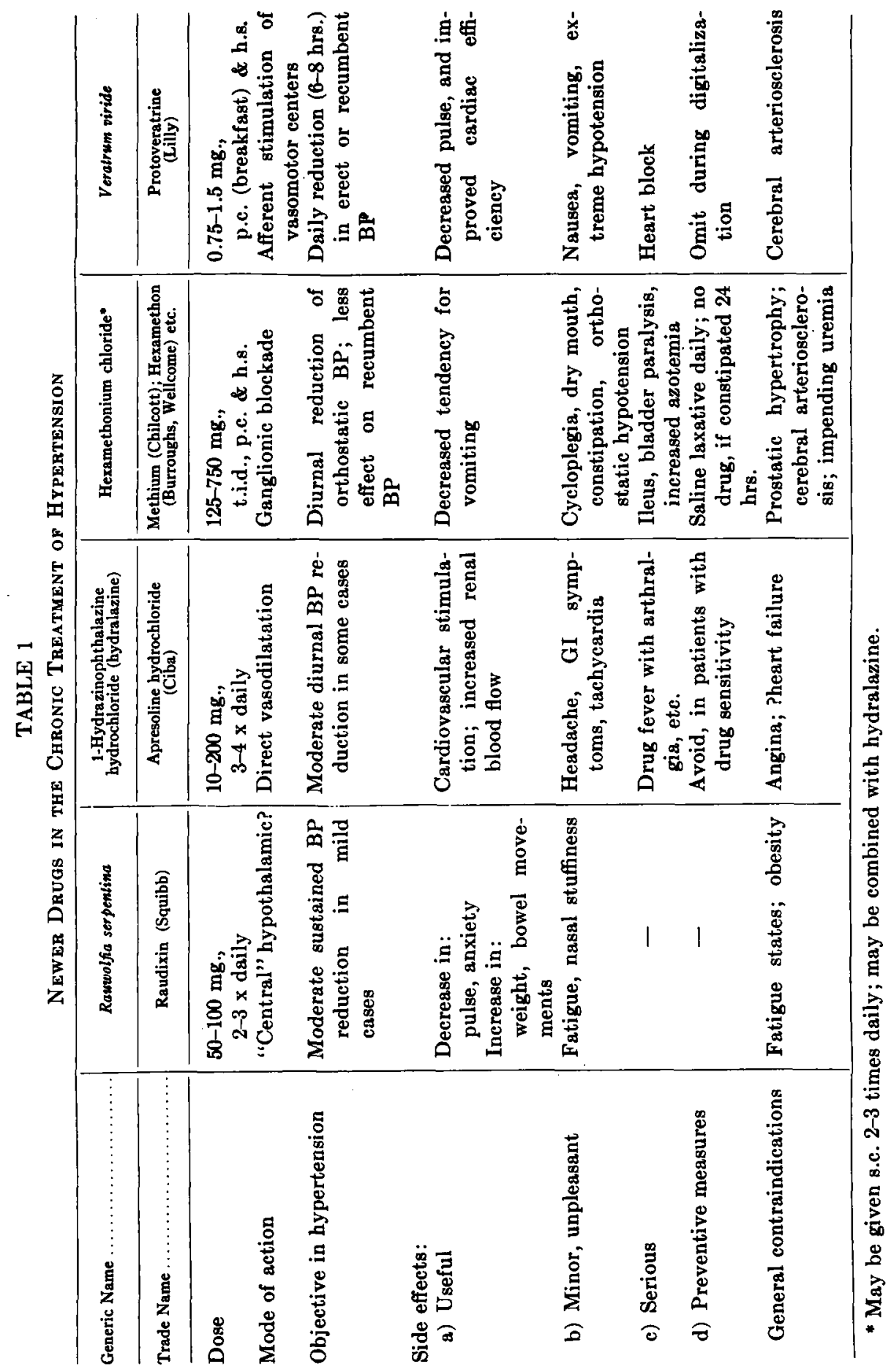




\section{Blood pressure moderately elevated; symptoms or complications present}

a. Angina. Salt restriction is frequently beneficial and, together with the accepted coronary vasodilators, is the treatment of first choice. In our hands the sedative and bradycardiac effects of Rauwolfia are sometimes helpful in managing angina pectoris. The tendency to obesity must be controlled, however. Oral hexamethonium is to be tried cautiously in order to avoid steep falls in blood pressure with the risk of precipitating coronary thrombosis. Moderate reduction in blood pressure often seems to relieve pain. Apresoline is contraindicated. Protoveratrine may be helpful.

b. Congestive failure. Conventional methods should be tried first, with emphasis on salt restriction (200-mg. sodium diet). If the blood pressure remains high and dyspnea or edema recur, hexamethonium should be used to control the daytime (ambulatory) blood pressure. Protoveratrine, if necessary, should be taken nightly for control of paroxysmal dyspnea. Hexamethonium must be used cautiously, as it may produce cumulative toxic effects in the face of the renal insufficiency which commonly accompanies congestive failure. Rauwolfia may help, especially if tachycardia is persistent, but it cannot be expected to lower the blood pressure markedly in most cases. Apresoline is probably contraindicated.

c. Cerebral thrombosis. Apresoline is theoretically the drug which is the most likely to improve cerebral blood flow. Despite this, I have seen thromboses occur in 3 patients taking Apresoline, without significant reduction in blood pressure. Measurements of the effect of the drug on cerebral resistance show only slight improvement (4). Consequently the choice of Apresoline for the treatment of cerebral thrombosis is not made with great conviction. Hexamethonium and Protoveratrine may lower the blood pressure too sharply and extend the thrombosis. Rauwolfia is probably not effective. Since reductions in pressure without concomitant cerebral vasodilation in an elderly person are likely to produce adverse effects, patients who have had thrombotic accidents as the only complication of hypertension should not be treated wtih depressor drugs.

d. Cerebral hemorrhage. The dramatic manifestations of this complication theoretically call for acute reduction in blood pressure. In practice, however, control of blood pressure during such an episode is difficult. Hexamethonium has acute effects but when the intracranial pressure is increased from bleeding, patients are extremely sensitive and may respond with severe hypotension. The drug should be given at a rate of $0.5 \mathrm{mg}$. per minute intravenously, with the patient in the sitting position and the blood pressure recorded every thirty seconds. Arfonad, a new vasodepressor, may prove more useful in such emergencies, as its effects are more transient and hence more controllable (7). When a patient presents himself with the residual effects of an old cerebral hemorrhage, a difficult therapeutic decision has to be made. In general, only symptomatic treatment is advised, because hypotensive therapy may precipitate a thrombosis; on the other hand, lack of hypotensive therapy may result in a hemorrhage. If the blood 
pressure is usually over 200 , moderate measures of treatment, such as salt restriction, Rauwolfia, Apresoline or even hexamethonium and Protoveratrine may be tried, provided the patient's general condition is good. Otherwise, it is best to avoid hypotensive drugs.

e. Hypertensive encephalopathy. By this term is meant the acute focal or generalized neurologic disturbances produced by severe hypertension in the absence of uremia or of chronic cerebral arteriosclerosis. When the latter complications can be ruled out, vigorous therapy is often dramatically effective in restoring consciousness and clearing residual neurologic symptoms. Hexamethonium should be given intravenously very slowly, as described previously, until the blood pressure is lowered to $170-180$ systolic.

f. Renal complications. Azotemia contraindicates almost all definitive drug therapy, because the prognosis is nearly hopeless anyway and vasodepression may lead to rapid progression of the uremia. There are two exceptions: 1) When the azotemia is pre-renal (i.e., cardiac, or due to dehydration) and responds to appropriate treatment, and 2) when other symptoms demand attention regardless of the renal status (i.e., convulsions, impending blindness from retinopathy, or refractory left ventricular failure). In the former instance hexamethonium should be very cautiously administered by subcutaneous injection beginning at $5 \mathrm{mg}$. every four to six hours and increasing by $5 \mathrm{mg}$. per dose until the blood pressure is lowered to a reasonable level. During this time, daily creatinine or blood urea determinations will indicate whether the uremia is improving or not. If not, treatment should be stopped immediately. Ileus, which may come on abruptly due to deficient renal removal of hexamethonium, is likewise a calculated risk in this situation. In the second instance, when uremia is clearly present, Protoveratrine or Apresoline is the treatment of choice. We favor the former in this situation, because the effect of Apresoline is often unpredictable. Considerable symptomatic relief may sometimes be achieved.

\section{SUMMARY}

The types of hypertension encountered in the elderly are described. Emphasis in treatment should be upon milder measures such as salt restriction, Rauwolfia derivatives and occasionally hydralazine. More vigorous methods such as sympathectomy, and the administration of hexamethonium chloride or Veratrum derivatives should only rarely be used.

\section{REFERENCES}

1. Perera, G. A., and Haklig, A. W.: Clinical characteristics of hypertension associated with unilateral renal disease, Circulation 6: 549-552, 1952.

2. Hoobler, S. W.; Manning, J. T.; Paine, W. G.; McClellan, S. C.; Helcher, P. O.; Renfert, H., Jr.; Peet, M. M., and Kahn, E. A.: The effects of splanchnicectomy on the blood pressure in hypertension, Circulation 4: 173-183, 1951.

3. Wilkins, R. W., and Judson, E.: The use of Rauwolfia serpentina in hypertensive patients, New England J. Med. 248: 48-53, 1953. 
4. Moyen, J. H.: Hydralazine (Apresoline) hydrochloride, Arch. Int. Med. 91: 419-439, 1953.

5. Moyer, J. H.; Snyder, H. B.; Johnson, I.; Mills, L. C., And Miller, S. I.: Results with oral hexamethonium alone and in combination with 1-hydrazinophthalazine (Apresoline) in the treatment of hypertension, Am. J.M.Sc. 225: 379-398, 1953.

6. Hoobler, S. W.; Corley, R. W.; Kabza, T. G., and Loyke, H. F.: Treatment of hypertension with oral Protoveratrine, Ann. Int. Med. 97: 465-481, 1952.

7. Sarnoff, S. J.; Goodale, W. T., and Sarnoff, L. C.; Graded reduction of arterial pressure in man by means of a thiophanium derivative (RO 2-2222), Circulation 6: 6373, 1952. 